\title{
Las controversias del pensamiento económico en el análisis de las actividades de maquila internacional
}

\author{
Alejandro Mungaray Lagarda \\ Dirección General de Asuntos Académicos \\ Universidad Autónoma de Baja California
}

\section{Resumen}

Este trabajo ofrece una reflexión acerca de los aspectos controversiales de la investigación económico-social como constante histórica en el desarrollo del conocimiento. La ubicación de fenómenos como el crecimiento industrial global vía maquiladora en un cuerpo teórico amplio es aún insuficiente. El enfoque considerado aquí como globalizante y más crítico es el de la nueva división internacional del trabajo. Se establece que la explotación capitalista no es privativa de este tipo de producción y por tanto no es cuestionable, al mismo tiempo que argumenta que son acciones que "han contribuido a desarrollar más ampliamente que nunca el crecimiento industrial en la frontera y en la transformación de todos los aspectos de la dinámica tradicional en la región".

Palabras clave: pensamiento económico, maquila internacional, crecimiento industrial global, división internacional del trabajo, liberalismo.

\begin{abstract}
This work is a reflection of the controversial aspects of socioeconomically research as a historical constant derived from the development knowledge. To locate the phenomena representing global industrial growth via maquila is still insufficient in a wide theoretical field. A new and more critical way to focus that is considered as a globalizing one is the new International division of work according to this research. It also states that capitalist exploitation is not exclusive of this kind of production; thus, it is not questionable, and at mean time it sustains that those are not actions that "have contributed to develop in a wider form of industrial growth in the border and to transform every aspect of the traditional dynamics of this region".
\end{abstract}

Keywords: economical thinking, international maquila, global industrial growth, international division of work, liberalism. 


\title{
LAS CONTROVERSIAS DEL PENSAMIENTO ECONOMICO EN EL ANALISIS DE LAS ACTIVIDADES DE MAQUILA INTERNACIONAL
}

\author{
Por: \\ Alejandro Mungaray Lagarda.
}

\section{INTRODUCCION}

Resulta fácil observar en lo extenso de la literatura económica y social, el enfrentamiento de las ideas en torno al estudio de cualquier fenómeno que intente comprender el hombre. Sin embargo, pese a lo atractivo que resultaría calificar esta situación como anormal, se requiere asimilar el hecho como normal y cotidiano. Yendo más lcjos, el aspecto controversial de las investigaciones en el campo de la economía y la sociedad, no es una característica meramente actual; tiene un carácter histórico. Casi se podría decir que ocurre desde siempre. Sin embargo, la diversidad de las ideas no puede ser resultado tan sólo de las elucubraciones intelectuales de los hombres, como de la diversidad de posiciones y visiones que a nivel social se manifiestan cotidianamente entre los distintos sectores sociales.

Este trabajo plantea algunas de las controversias más relevantes en la historia del pensamiento económico, como antecedente que permite entender la gran diversidad de enfoques de los investigadores en su quehacer cotidiano en torno a objetos de análisis concretos; en este caso las actividades de maquila o subcontratación internacional, que aquí son tratadas en términos generales sin que sea posible afirmar que estén agotadas.

Por áltimo, la ubicación de los análisis de maquiladoras en cuerpos teóricos más amplios, es todavía insuficiente, sobre todo por la gran producción intelectual existente y el limitado acervo de la misma que aquí se comenta. Sin embargo, es de suponer que los resultados aquí expuestos poco variarían con la inclusión de nuevos materiales, que a lo más proporcionarían líneas de interpretación adicionales.

\footnotetext{
- Director General de Asuntos Académicos, Universidad Autónoma de Baja California.
} 


\section{CONTROVERSIAS IMPORTANTES DENTRO DEL PENSAMIENTO ECONOMICO}

\section{Ricardo-Marx}

Dentro del campo del pensamiento cconómico, son muchas las variaciones en torno a un mismo tema: el qué producir, cómo producir, para quién producir, e innovando un poco, cómo se distribuye lo producido. Lo curioso en estas variaciones es que pese a estar claras las respuestas, se cubren con tecnicismos.

Con lo anterior se quiere decir que si se considera el contexto capitalista que impregna las relaciones de producción y distribución que organizan la sociedad, qué producir se responde con mercancías; cómo producir se responde con medios de producción y organización capitalista del trabajo; para quién producir se contesta con el mercado y; cómo se distribuye lo producido se responde con los sectores de la sociedad que participan en la producción. Esta idea, que no es novedosa ni original, prevalece en el pensamiento económico desde el siglo pasado, cuando David Ricardo planteaba su idea de cómo podía funcionar la sociedad y que su producto creciera a partir de reconocer la existencia de clases sociales y de un cierto mecanismo automático de distribución del ingreso entre éstas, que hace que unas sean más productivas y/o progresistas que otras. Al establecer la relación entre clases sociales, agentes de la producción y formas en que participan en la distribución de la riqueza, precisaría que la renta que reciben los terratenientes, se destina principalmente al consumo de bienes suntuarios; los salarios de los trabajadores se destinan al consumo exclusivo de bienes de primera necesidad para su subsistencia, y los beneficios, dando por hecho el frugal consumo de los capitalistas, se asignan en su mayor parte a la produccion de bienes; es decir, se reinvierten permanentemente en actividades industriales que dan lugar a procesos de acumulación de capital que posibilitan el crecimiento económico. Como se ve, el crecimiento sostenido se logra debido al tipo de distribución observado, aunado al consumo característico de las clases (Pasinetti, 1980: 17-8 y 1983 :35). 


\section{CUADRO SINOPTICO}

\begin{tabular}{llll}
\hline $\begin{array}{l}\text { Clases } \\
\text { sociales }\end{array}$ & $\begin{array}{l}\text { Agente econ. } \\
\text { que aporta a la } \\
\text { producción }\end{array}$ & $\begin{array}{l}\text { Forma que asu- } \\
\text { me el ingreso } \\
\text { que perciben }\end{array}$ & $\begin{array}{l}\text { Destino } \\
\text { principal } \\
\text { de ingresos }\end{array}$ \\
\hline $\begin{array}{l}\text { Terratenien- } \\
\text { te }\end{array}$ & Tierra & Renta & Bienes suntuarios \\
$\begin{array}{l}\text { Capitalista o } \\
\text { empresario }\end{array}$ & Capital & $\begin{array}{l}\text { Ganancia o } \\
\text { beneficio }\end{array}$ & Reinversión \\
$\begin{array}{l}\text { Trabajadores } \\
\text { Trabajo }\end{array}$ & Salarios & $\begin{array}{l}\text { Biencs de } \\
\text { consumo no } \\
\text { duraderos }\end{array}$ \\
\hline
\end{tabular}

Ahora bien, los empresarios capitalistas son los agentes promotores del crecimiento cconómico porque al reinvertir son los únicos que acumulan, es decir, porque son los únicos capaces de invertir. Sin cmbargo, esta capacidad no es fortuita; csto es, no resulta tan sólo de la aplicación productiva de los bencficios obtenidos, como de una distribución previa de los instrumentos de producción entre los individuos, y de éstos en las distintas ramas de producción resultantes de la creciente división social del trabajo. Ello podría remitir a aquellos métodos idílicos de la acumulación originaria de capital, que de idílicos no tuvieron más que lo irónico del lenguaje de Marx. Por tanto, un enfoque crítico no es precisamente aquel que parte de la crítica de la mala distribución del ingreso entre los sectores sociales, según el esquema de Ricardo, sino aquel que critica la distribución del ingreso proveniente dela propiedad (Marx, 1977a: 16-17). Así, todas las formas y"relaciones de distribución aparecen sólo como reverso de los agentes de producción. (En otras palabras), la organización de la distribución está totalmente determinada por la organización de producción. La distribución es ella misma un producto de la producción" (Ibid: 15). La distribución capitalista presupone la propiedad capitalista.

De esta forma, las aseveraciones ricardianas de que los únicos capaces de impulsar la acumulación de capital son los empresarios, son incuestionables. Sin embargo, la consideración marxista hace la diferencia de que aunque el empresario sea el elemento dinámico del proceso de acumulación capitalista, ello no le convierte, desde ninguna 
óptica y/o perspectiva, en el generador de la riqueza que se produce en la sociedad, mucho menos en el responsable directo de su potencialidad. Así, Marx introduce el concepto de plusvalía que revoluciona el pensamiento económico desde sus raíces más antiguas y hasta nuestros días. Este concepto se refiere a la capacidad que tiene el capitalista para obtener una mayor cantidad de trabajo que la que pagó. Esto lo logra mediante el aprovechamiento del régimen de trabajo asalariado y de la contratación de los obreros por un salario durante una jornada de trabajo establecida socialmente, donde éstos (los obreros) producen más valor de lo que ellos mismos significan en términos de susalario. En otras palabras, aunque el elemento dinámico del proceso de acumulación o crecimiento capitalista parezca ser el empresario que posee el capital que organiza la producción, es el trabajador quien en última instancia produce la riqueza. Con esto se ilustra que "la producción de plusvalía (...) es la ley absoluta de este sistema de producción" (Marx, 1977b: cap. XXIII).

Como se ve, las diferencias entre los pensamientos de Marx y Ricardo son radicales. Entanto que Ricardo propone, bajoun esquema de clases sociales y las formas que asume su ingreso-gasto, que sólo los empresarios capitalistas son capaces de organizar la producción social, Marx, sin negarlo, scñala que esta capacidad no es fortuita ni tiene como fin exclusivo el lograr el crecimicnto económico. Añade que si los capitalistas son los únicos capaces de acumular capital, es porque éstos son ya dueños de él y lo han puesto a funcionar empresarialmente. Ello implica la organización de la producción por la vía del contrato salarial de los trabajadores; ́sos que Ricardo señala tan sólo como un elemento de la demanda, y que en Marx son sometidos a una disciplina de cxplotación que permite a los progresistas empresarios de Ricardo impulsar el crecimiento económico de la sociedad.

Si se vuelve a la pregunta inicial, se podrá notar que la existencia de visiones distintas de un mismo proceso, como lo es el de la acumulación de capital, dan origen a controversias donde cada protagonista establece sus líneas de crítica y desmitificación del pensamiento contrario, resultando que la socialización de la polémica se allega tanto de referentes empíricos como de adeptos, impregnándose de csta manera en el contexto de la sociedad.

Así queda planteada una gran línea de controversias dentro del pensamiento cconómico, aún cuando sea tan sólo en uno de sus aspectos: la base de la acumulación de capital. 
Liberalismo-nacionalismo: argumentos teórico-políticos.

Otra línea de controversia más contemporánca y con mucha actualidad práctica, se desarrolla en tornoal proyecto más conveniente para organizar las sociedades modernas: por un lado se perfila el proyecto ncoliberal con fuerte inspiración neoclásica, en cuanto que sostiene que el equilibrio de la economía se puede lograr mediante mecanismos automáticos de las fuerzas de mercado; por otro, se perfila el proyecto democrático nacionalista de fuerte inspiración keynesiana, donde se pone gran énfasis en la intervención del estado en la economía para regular o equilibrar aquellas variables macroeconómicas que los mecanismos automáticos de las fuerza del mercado no pueden hacer. Comoquiera que sca, lo que en realidad parece estar en discusión es la intensidad con que debe participar el estado en la economía, así como la dirección en que debe actuar a través de la política económica. De ninguna manera se discute su intervención, sino tan sólo el rumbo que ésta ha de tomar en beneficio de los diferentes sectores capitalistas locales. Sin embargo, en la medida en que el desarrollo capitalista ocurre siguiendo su propia dinámica y al margen de la voluntad de las unidades economicas individuales, el control de las fuerzas y desequilibrios que desata han intentado ser controlados a través de la planificiación. Con esta preocupación, los economistas principalmente, se han visto en la necesidad de elaborar permanentemente planes y/o modelos donde, mediante la conjugación de lo que a su juicio consideran las más relevantes variables de comportamiento de la sociedad, elaboran normas deseables de comportamiento para la economía. Conforme ha ido pasando el tiempo, la elaboración de esos modelos se ha ido sofisticando a la vez que complicando, debido fundamentalmente a la necesidad de incluir permanentemente nuevas variables económicas así como de otro tipo que, sin ser específicamente económicas, influyen sobre el comportamiento de la economía (Carrillo Huerta, 1978:96). De otra forma, la parcialidad de los modelos se ha ido corrigiendo permanentemente.

En este contexto surge la disputa sobre la forma de lograr el equilibrio social que permita el crecimiento y a la vez lo propicie. Por una parte está la posición de laissez-faire que sostiene que los problemas económicos y sociales de una nación "tendrán solución una vez que el sistema económico global funcione óptimamente, porque desde su perspectiva se trata en lo fundamental de imperfecciones que son el producto de las rigideces impuestas al mercado. De esta forma 
(...), el centro de la reflexión programática (...) es ocupado por los grandes agregados económicos, tal y como aparecen en el mercado" (Cordera y Tello, 1981:3). Oferta y demanda deben regir el curso y procurar el equilibrio de la economía a través de los mecanismos automáticos del mercado; ellosupone la intervención de un elemento invisible y por tanto inexplicable que regula el empleo, la inversión, el interés, etc. (Smith, 1979:56).

Por otra parte, y partiendo de que "la economía de mercado tiende a dirigir la producción y la distribución hacia el interés público" (lo que procura no negar la importancia del mercado), se plantea la cuestión de "si la planeación (realizada por el estado) no podría hacerlo mejor" (Lewis, 1975:37). Como se señaló antes, la discusión gira en torno a qué parte del control sobre la economía puede ser invisible y dejada al libre juego de las fuerzas de mercado, y qué parte debe ser visible y organizada por el estado. Ahora bien, el hecho de que el estado funcione como regulador del desarrollo, sujeto a los lineamientos fundamentales de la economía de mercado pero sin depender de la acción reguladora de sus mecanismos automáticos, sirve de indicador acerca de la existencia de diferentes fracciones capitalistas con intereses tan contrapuestos, que a la vez que requieren de un elemento mediador (que sea a la vez integrante) que les permita avanzar hacia el objetivo común, absorba gastos de interés público que, por no ser rentables en el sentido estrictamente económico, no están dispuestos a solventar.

Por lo regular, la burguesía neoliberal se ha caracterizado por contraponerse férreamente a los intereses nacionalistas. Hasta cierto punto, podría decirse que posee un alto espíritu internacionalista y contribuye en alto grado a expandir las formas de produccion y vida propias del capitalismo. Sin embargo, como los empresarios capitalistas no defienden más intereses que los suyos propios, perseguir incrementar subeneficio a costa de afectar el ajeno, les ha acarreado la oposición de las burguesías nacionalistas, sobre todo porque la capacidad de competencia de los productos de estas últimas se ha caracterizado por ser permanentemente menor a los de las burguesias internacionalistas neoliberales, provenientes de países con mayor grado de desarrollo de sus fuerzas productivas internas y con una capacidad financiera mayor que les da capacidad de movilización mundial. 


\section{Liberalismo-Nacionalismo: evolución de la controversia en el pensamiento económico latinoamericano.}

La controversia liberalismo-nacionalismo sí tiene nacionalidad, aunque es frecuente que la adopción de los postulados de una u otra posición la transgreda, porque en la medida que las ideas se van asimilando en cl cuerpo social, éstas son adoptadas como propias por los entes individuales y, al perder su nacionalidad, se universalizan. Esto explicaría la coexistencia de los pensamientos señalados en contextos nacionales específicos.

En América Latina, hasta antes de la crisis de 1929, prevalecía la idea de que el desarrollo económico sería realizado en virtud de la magnitud del financiamiento externo, que a través de inversiones directas en los sectores productivos realizaran los empresarios estranjeros. Con el "crack" se pudo comprobar la enorme vulnerabilidad del desarrollo capitalista latinoamericano, sostenido en el impulso de las economías de exportación al mercado mundial en función de la demanda de materias primas de los empresarios de los países industrializados. Esta situación prosiguió hasta la segunda guerra mundial, en que una industrialización espontánea tuvo lugar en América Latina debido a la creciente demanda de bienes de consumo no duraderos (con cierta transformación industrial) provenientede las potencias beligerantes. Tras esta experiencia "espontánea", en 1948 la CEPAL institucionalizó su funcionamiento como promotora de la industrialización latinoamericana, adaptándose más que nada un proceso que, aunque lento y sin organización, ya venía dándose de suyo.

En el contexto de la controversia señalada, la idea cepalina de planificar el crecimiento industrial no se disocia de la concepción keynesiana del control estatal de la economía sin afectar las estructuras de mercado capitalistas. Aún más, en sus inicios, esto es, hasta principios de los sesenta, la idea cepalina del crecimiento industrial desde la perspectiva nacionalista contenida en el modelo de sustitución de importaciones, sólo concebía la inversión extranjera bajo la forma de financiamientos gubernamentales a través de las instituciones creadas para tal efecto a partir de la segunda guerra mundial (BID, AID, etc). Así en el primer informe de la CEPAL, en 1949, se lanza la convocatoria de impedir que los precios arrebaten los frutos de la productividad, dada la estructura económica internacional, e impulsar la industrializacion, creando una estructura yun marco proteccionistas. Y aunque los hombres por símismos suclen 
ser menos importantes que la obra en que participan, en el surgimiento y desarrollo de la CEPAL fue sumamente importante la presencia de Raúl Prebisch quien con ello se convirtiera en uno de los máximos exponentes del pensamiento estructuralista latinoamericano, que tanta influencia tuviera sobre elfuncionamiento de la política económica de varios países de la región durante el período que va de fines de los cuarenta a principios de los sesenta. En resumen, para Prebisch la problemática central de las economías latinoamericanas descansa en la flagrante y desigual distribución del ingreso prevaleciente, así como enel deterioro de los términos deintercambio que tanto afectan el desarrollo de la empresa capitalista latinoamericana.

De esta forma, Prebisch no impugna el juego de mercado, aunque sí las estructuras que están detrás del mismo. Con ello reconoce la existencia de un complejo mecanismo de división internacional del trabajo donde, dadas las características del desarrollo capitalista desigual de los distintos países, los países latinoamericanos juegan un dramático papel.A la vez, con esto reconoce también que el mercado es ineficiente "para resolver las flagrantes desigualdades en la distribución del ingreso (ya que ésta) se explica por un juego de relaciones de poder que surge de las mutaciones de la estructura social" (Prebisch, 1980:VIII). Con todo, el interés de Prebisch no radica en la eliminación del mercado; por el contrario, persigue mejorar su funcionamiento mediante el mecanismo de la planificación para regular la distribución del ingreso, ya que "el mercado refleja los fenómenos estructurales y funciona bien o mal según sea la distribución (Ibid.:IX).

De lo anterior se deduce que, como la división internacional del trabajo se establece en función de la estructura productiva determinante en los diferentes países, el papel que corresponde a las "economías periféricas", debido al carácter especializado y heterogéneo de su estructura productiva, es el de producir y exportar materias primas y alimentos a todo el sistema como "el instrumento para conseguir (...) las importaciones de bienes manufacturados" requeridos por la demanda interna (Rodríguez 1980: 27,309). De esta forma, el desarrollo de estas economías descansa en el crecimiento de la producción del sector primario y de las importaciones y, dada la coexistencia con economías con un crecimiento más firme basado en el desarrollo de su industria pesada, se genera una tendencia de subordinación y dependencia a través de las relaciones de intercam- 
bio de las burguesías latinoamericanas con respecto a las de los países capitalistas más desarrollados.

Desde otro punto de vista, la tendencia señalada no necesariamente genera una situación de estancamiento en el aparato productivo como pretende hacerse creer. Más bien caracteriza una situacion típica de los países con menores niveles de productividad e ingreso per cápita con respecto a otros, que buscan las mejores vías de fomentar un desarrollo capitalista y con ello el de las burguesías nacionales, pues en estas condiciones, participan económica y políticamente subordinadas a las burguesías extranjeras que ejercen su dominio a través de los mecanismos del comercio internacional de bienes y capitales y de la inversión extranjera directa, propios del proceso liberal.

Es entonces cuando surgen esas aparentes coincidencias que vienen a explicarnos cómo es que existen tradiciones dentro del pensamiento económico. En efecto, a partir de lo señalado anteriormente, no debe extrañar que el desarrollo económico en el capitalismo se conciba en Prebisch, al igual que en Ricardo, como un proceso de acumulación y progreso técnico que tiende a elevar la productividad por hombre ocupado y que, al lograr el incremento del ingreso per cápita, beneficie el nivel de vida de la población. Así, el consumo frugal de los empresarios que en Ricardo se presenta como una cualidad natural, en Prebisch se presenta como una cualidad en potencia que hay que estimular para lograr una mayor acumulación de capital y una mejor distribución del ingreso. Esta se logra al reducir, mediante mecanismos de política económica, el consumo de los estratos superiores de la estructura social, empujándose a invertir en nuevos proyectos de inversión. Consecuentemente, a nivel social hay cambios en la composición orgánica del capital y en la estructura productiva, a la vez que una mayor generación de empleos y por tanto, de ingresos.

Las enseñanzas que obtuviera Prebisch de Marx y Ricardo, le lleva a adoptar la lógica de la acumulación de capital desde la perspectiva de la composición orgánica de capital creciente, y pensar que sus efectos, en el sentido de que conforme avanza la acumulación de capital la inversión tiende a destinarse más a consumir medios de producción que fuerza de trabajo, pueden ser contrarrestados con medidas de política económica. Así, la tendencia contradictoria en Marx de que a mayor acumulación de capital y por tanto mayor crecimiento de la composición orgánica de capital, corresponde una disminución de la tasa de ganancia, es según Prebisch, fácilmente contrarrestable para así 
lograr la distribución del ingreso que planteaba Ricardo a fin de promover el crecimiento económico.

El crecimiento económico sostenido fue un hecho en laseconomías latinoamericanas, pero las tendencias contradictorias de la industrialización enunciadas por Marx no pudieron ser contrarrestables con medidas de política económica; el desempleo hoy está por los cielos y la tasa de rentabilidad por los suelos.

Y se habla de "hoy" porque son resultados de un proceso de largo plazo. Sin embargo, en sumomento Prebisch fue un visionario, cuando reafirmaba su preocupación por incrementar la productividad como la única forma de impulsar la acumulación y mejorar el nivel de ingresos a través de lograr mayores ganancias, dentrode lo intrincado de la competencia, mediante la modernización de la estructura productiva imperante.

La modernización no ocurrió y por añadidura lo demás tampoco. Las estructuras proteccionistas que requería la industrialización se fundieron con el proceso de acumulación de capital de las economías latinoamericanas, hasta que el agotamiento de esta fase del proceso de acumulación causó estragos a nivel social. Entonces hubo cambios: el neoliberalismo entró en acción e impuso su modelo de economía frente a los declinantes proyectos nacionalistas (Villareal, 1983). La "manu militari" se extendió por el continente, siendo los casos más visibles del nuevo modelo neoliberal el brasileñoy el chileno (Briones, 1979). Sin embargo, aunque los fracasos de la política económica neoliberal están a la vista, como también lo están en el caso de los modelos nacionalistas, parece ser que el problema de quién controla la economía es un problema insignificante si se considera que a través de las tendencias internacionalistas de los modelos neoliberales, el patrón de acumulación en las economías latinoamericanas, vale decir, la modalidad de reproducción social del capital, se está transformando. No obstante, las economías de modelo neoliberal han tenido que vivir doblemente el peso de las crisis. Primero, impulsada por la nueva política económica para recomponer la supuestamente nefasta actuación estatal de corte populista; y después por el fracaso de la política económica neoliberal que no pudo evitar los embates de la crisis que se desencadenó a todo lo largo y ancho de la órbita mundial, mostrando a todos que el problema de la crisis es un problema de agotamiento de los patrones de acumulación, de agotamiento de las formas que asume la reproducción social y de la consiguiente caída de 
la tasa de ganancia, y no de mal funcionamiento de la política econ6mica como se ha querido hacer creer con insistencia.

Resumiendo lo hasta ahora dicho respecto a las controversias señaladas, el aspecto controversial Ricardo-Marx aquícsbozado, radica en que mientras Ricardo tiene como objetivo explicar por qué el empresario es el único capaz de impulsar el capitalismo sin mayores problemas, definiciones ocuestionamientos, Marxhace precisamente lo contrario; porque él busca una explicación crítica de por qué los empresarios son los únicos capaces de impulsar el capitalismo y cómo utilizan el trabajo asalariado para tal fin. Se podría decir que lo que Ricardo hace es reconocer una realidad palpable $y$ visible organizándola sin mas para explicar el capitalismo, en tanto que Marx, partiendo de la misma realidad concreta y visible, profundiza en su presencia.

Por otra parte, en la controversia liberal-nacionalista, la polémica consiste en definir si el cuerpo teórico neoclásico o el neokeynesiano es el más apropiado para explicar y darle salida a los problemas del capitalismo contemporáneo agudizados por la crisis. Lo que no se advierte es que la crisis del capital es a la vez la crisis del pensamiento económico convencional, y que la intensificación de la disputa en la actualidad es el reflejo de la urgente necesidad que se tiene por articular un cuerpo teórico modernizado que ayude a resolver los problemas que vive el capitalismo (González y López, 1984:147-171). Por último, los postulados más generales de los primeros serían: cese al proteccionismo estatal de la industria; liberación de los precios; libre acceso a la inversión extranjera; control de la inflación, del salario y del gasto público en un sólo paquete; etc., en tanto que los de los segundos serían la disminución de la independencia respecto al exterior; una mejor distribución del ingreso; protección a la planta industrial nacional, etc. Por supuesto que ésta es una forma muy simplificada de presentarlos, pero que con fines de comparación es sumamente útil.

\section{LAS MAQUILADORAS Y LOS ENFOQUES DEL PENSAMIENTO ECONOMICO}

Uno de los fenómenos más controversiales dentro del terreno del pensamiento económico, especialmente cuando se le circunscribe en el context o fronterizo, escl de la instalación de plantas maquiladoras de las ciudades fronterizas del norte de México, pues durante los 20 
años en que las maquiladoras han estado realizando formalmente operaciones productivas en cl país, han sido objeto de una abundante discusión que sin ser eminentemente teórica, ha sido realizada principalmente en los marcos de las ventajas y desventajas que representan para México (Kuesel, 1986:2). De otra forma, en el marco de la controversia liberal-nacionalista que consiste en definir si el cuerpo teórico neoclásico o el keynesiano es el más apropiado para explicar y proponer salidas a los problemas del capitalismo contemporáneo agudizados por la crisis.

Las posiciones que resaltan las ventajas de las maquiladoras en los países como el nuestro, curiosamente provienen de los dos puntos de vista, aunque por motivos diferentes: en primer lugar se encuentra la posición nacionalista que de entrada afirma que las maquiladoras son elementos de desarrollo nacional. Esta afirmación se realiza desde la óptica de las ventajas que representan para la nación en términos del logro de algunos de los postulados nacionalistas, ya sea en términos del abatimiento del desempleo; de su aportación a la balanza de pagos; de su efecto multiplicador sobre la actividad económica regional al ensanchar, vía derrama de ingresos, el nivel de demanda efectiva; y de su participación en la capacitación industrial del trabajo. Este razonamiento de tipo neokeynesiano, deriva del interés puesto "en los efectos que la distribución del ingreso tiene sobre el desarrollo económico y viceversa, los que el desarrollo económico tiene sobre la distribución del ingreso" (Carrillo Huerta, 1978: 93). Ahora bien, el razonamiento lógico que da razón de ser a estas posiciones es el siguiente: la inversión por maquiladoras, al significar inversión productiva de procedencia extranjera que se desarrolla en función de actividades productivas extranjeras, pero que legalmente está parcialmente imposibilitada para concurrir directamente con su producción en el mercado nacional, genera divisas por servicios de transformación y genera empleos que a la vez que abaten el nivel de desempleo, proporcionan poder de compra a un sector relativamente importante de la población, principalmente de la frontera norte del país, donde el crecimiento de la población es muy superior al de la actividad económica. Esto beneficia al sector comercial de la región, que aprovecha el mayor mercado que significa la derrama de ingresos por conceptode sueldos y salarios paravender más productos. El sector industrial se puede beneficiar, o bien colocando sus productos en el mercado regional a través del comercio local, o bien sustituyendo con sus productos algunos de los insumos y materias primas que 
utilizan este tipo de plantas. Si csto se observa en sentido acumulativo, las maquiladoras, al contribuir al mejoramiento del nivel de vida de la sociedad, contribuyen a mantener la estabilidad que requicrc un país para crecer económicamente (Bermúdez, 1966; Alcalá, 1969: 960-964; Hunt, 1970; Rivera, 1971; Rivas Sosa, 1973; S.I.C., 1975; Urquidi y Méndez, 1975:149-174; Torres, 1976:1406-1413; BNCE, 1978; Köning, 1979; Banamex, 1979:676-679 ; 1981a; 1981 b:283-290; Teutli, 1981:15; Martínezdel Campo, 1983: 146-155; FONEI, 1984; Davis, 1985; Rivas, 1985: 1071-1084; Mungaray, 1985a: 31-35; Lopez Lucio, 1985: 14 ; Puente, 1986: 39-41).

En segundo término se encuentra aquella posición que estimula la instalación de las maquiladoras, pero desde la perspectiva de las ventajas que representa para los empresarios de donde provienen las actividades de maquila. Estos apelan al libre juego de las fuerzas del mercado para justificar que la inversión no debe tener restricciones, mucho menos cuando ayuda a resolver problemas nacionales. Acuden también al encanto de autodesignarse como impulsores y promotores del desarrollo económico de los países relativamente menos desarrollados como son los de Asía, Africa y América Latina, gracias al riesgo que corren al invertir en países tan inestables, ya que de otra manera su desarrollo económico sería más lento. Esta posición prevalece fundamentalmente en los círculos oficiales y empresariales de los países industrializados y también, aunque con menor fuerza, en los círculos académicos. (Norris and Elliot, 1969; Steiner, 1971:2-5; Briggs, 1973; Reynolds, 1980: 11-39; Grunwald, 1983: 2123-2152; Dillman, 1983:3158; Appleyard, Field and Tower, 1985: 62-65; Reyes, 1986: 52; Drucker, 1987: 3-16; Grunwald, 1987: 27-46).

Por otra parte, se configura una variada corriente de opinión crítica en la cual el logro de los objetivos de un desarrollo nacionalista democrático, autónomo e independiente de los designios del capital extranjero a través del Programa de Industrialización Fronteriza, se cuestiona desde diferentes y muy variadas perspectivas. Esta corriente crítica se caracteriza por no ir más allá de la crítica de los planteamientos gubernamentales y de la injusticia laboral en las maquiladoras cometida por el capital extranjero, sin atender el problema derivado de la creciente presencia del capital nacional, lo que viene a darle un carácter de vigilante parcial del logro de los objetivos del desarrollo nacional, sin más perspectiva crítica que la de intentar arreglar lo descompuesto. Se puede decir que no es una crítica que profundice en la búsqueda de una explicación en torno al significado esencial 
de la presencia de las maquiladoras y sus tendencias y las cucstione en tal sentido. Aquí caben múltiples variantes que se pueden sintetizar como sigue:

a) Los empleos generados son incstables y en su mayor parte son ocupados por mujeres (Bustamente, 1975: 183-215 ; Murayama y Muñoz, 1975; Escamilla y Vigorito, 1979; García Moreno, 1979; Gambrill, 1979a; S.F.; 1980: 5-7; 1981 a: 7-60; 1984; Savane, 1980: 861866; Vera, 1980: 9-11; Carrillo y Hernández, 1982; Fernández y De Bucn, 1984: 340-368; Anderson, 1984: 87-95; Iglesias, 1984).

b) La incorporación de la mujer al régimen del trabajo asalariado, acarrea la desintegración familiar y la corrupción de los valores sociales. (Bustamante, 1975: 183-215; Muller, 1976; Gambrill 1979a; 1979b: 5 6; 1980: 5-7).

c) La escasa integración de insumos ymaterias primas a los procesos de maquila, contradice los objetivos de su instalación e implica una desvinculación de la economía fronteriza con el resto de la estructura productiva nacional. (Bustamente, 1975: 183-215; Torres Gaytán, 1979: 5-12; Fernández, 1980; Tamayo, 1980: 191-192; Gambrill, 1981a: $7-$ 60; Suárez Villa, 1982: 1129-1132; Fernández y De Buen, 1984: 340 36; Ortfz, 1986: 19-21; Oppenheim y Pedroza, 1987: 28-37).

d) La capacitación industrial de la fuerza de trabajo es muy relativa debido a la imposibilidad de integrarla a una planta industrial nacional concentrada y con poco dinamismo (Bustamante, 1975: 183-215; Fernández, 1980; Fernández y De Buen, 1984: 340-368; Verkoren y Hoenderdos, 1986: 19).

e) Los beneficios fiscales y laborales concedidos a estas plantas, son excesivos en comparación con los beneficios que ellas dejan al país. (Enriquez, 1971; Bustamante 1975: 183-215; Fernández, 1980; Fernández y De Buen, 1984: 340-368; Anderson y Frantz, 1985: 369378).

f) Sus actividades son muy inestables debido a que su funcionamiento depende de la producción de los países capitalistas más desarrollados (casi todos los trabajos).

g) Las relaciones de dependencia que se generan respecto al imperialismo norteamericano en el caso de México, trunca la posibilidad de un desarrollo autónomo que favorezca al desarrollo del empresario nacional. (Baird \& Mc Caugham, 1975; Bustamante, 1975: 183-215; Teutli, 1976: 9-40; Xirao y Díaz, 1976; Trajtenberg, 1978; García Moreno, 1979; Vaitsos, 1979: 149-192; Toro,1979: 34-41; Juárez, 1979; Torres Gaytán, 1979: 5-12; Fernández, 1980: 201-210; 
R. Fernández, 1980; Carrillo y Hernández, 1982b; Villarespe y Rodríguez, 1981; Calderón,1981: 62-71; Báez, 1983:9-11;Blanco, 1983: 13-16; Pourdanay, 1986: 11; Verkoren y Hoenderdos, 1986: 19; Sklair, 1987: 18).

h) Las condiciones en que se desarrolla el proceso de trabajo son sumamente degradantes y peligrosas y en muchos casos son la causa de que estén operando en México. (Talbot \& Hricko, S/F: 8;Villarespe y Rodríguez, 1981; Iglesias y Alarcón, 1981: 6-7; Carrillo, 1984b;Alvarez y Mungaray, 1986: 168-185).

Otro tipo de enfoques más globalizantes y a la vez más críticos, resaltan a las maquiladoras no en torno a las ventajas y/o desventajas de su instalación en una nación, sino en el contexto de la configuración de una nueva división internacional del trabajo, o como una de las formasque asume la internacionalización del capital bajo la modalidad específica de la internacionalización de procesos productivos, dándose un enorme salto en el terreno de la explicación científica del fenómeno en cuestión, ya que se sale de los marcos estrechos de la controversia sin fin ni perspectiva, para ubicar la problemática de las maquiladoras en el marco de las tendencias de la reestructuración capitalista, de tal manera que exista un hilo explicativo de la presencia de estas plantas en países como los nuestros. (Fajnzylber, 1973: 115-149; Duncan, 1976: 4-25; Bustamante, 1976:23-24; Froebel, Heinrichs y Kreye, 1978: 831-836; 1981: 580; Trajtenberg, 1978; Kampetter, 1979; Vaitsos, 1979: 149-192; Balli, 1980; R. Fernández, 1980: 201-210; Arriola, 1981; Minian 1981: 133; Chávez 1981; Villarespe y Rodríguez, 1981; Calderón, 1981: 62-71; Hansen, 1981: 4-12; Mungaray, 1983a: 40-44; 1983b: 231-253; 1983c: 19-27; Opalin 1984; Suárez Villa, 1985: 54-108; Storper y Walker, 1984: 19-47; Haring, 1985: 146; González Aréchiga, 1987: 46-50).

\section{CONCLUSION}

Como se ve, discutir en torno a si las maquiladoras son clementos de desarrollo o dependencia nacional, brinda la impresión ac estar dando vueltas alrededor de un gran círculo sin salida; abundar sobre la explotación que ejercen cotidianamente sobre una fuerza de trabajo barata, sumisa y desorganizada, no proporciona ningún elemento nuevo ni peculiar que permitiera distinguir este tipo de industrias de otras, aunque defender csto último resulte cstratégico ante quienes defienden sin restricciones la benevolencia de los empleos que 
generan. Por tanto, aunque resulte importante no perder de vista los elementos que permiten explicar desde todos los ángulos posibles la presencia de las maquiladoras en países con menor grado de desarrollo económico, más importante resulta recuperar la totalidad del fenómeno, de tal forma que sea posible su estudio en una perspectiva histórica que permite vislumbrar algunas tendencias significativas.

En tal sentido, no importa tanto discutir si las maquiladoras son factores de desarrollo nacional o si son factores de un desarrollo dependiente por provenir la inversión y/o los contratos de elementos extranjeros y ajenos a los intereses nacionales. Estos esfuerzos analíticos, en el mejor de los casos, lo único que dejan en claro es que el desarrollo industrial de la frontera norte de México, que es un hecho, se contempla desde perspectivas distintas sobre cómo es y cómo debiera ser el desarrollo industrial de la región.

Aquí, pasando por alto esta discusión eterna y en círculo, aunque sin desaprovechar muchos de los resultados de investigación que se han aportado, se proponen elementos indicativos de que con la industrialización vía maquiladoras, ha ocurrido un proceso de profundización capitalista en la región que poco tiene que ver con la nacionalidad del capital que la engendra, y mucho con las necesidades de reproducción de capitales individuales, atomizados y enfrentados entre sí, en un contexto competitivo que exige mayores niveles de productividad como base objetiva de la competitividad y, por tanto, de la ganancia.

Estos elementos indicativos son los que se pudiera llamar, en términos empresariales, factores de localización industrial, y más que plantearse en términos de atractivos preexistentes donados y puestos en bandeja de plata por un estado débil e inconsciente, se plantean como factores de competencia estatal ampliamente aprovechados tras una actividad industrial sin precedente, donde participan con objetivos e intereses comunes que a la vez los contraponen en el largo plazo, industriales extranjeros y mexicanos cuya acción ha contribuido a desarrollar más ampliamente que nunca el crecimiento industrial en la frontera, que de manera importante ha influido en la conformación de una nueva base regional de desarrollo capitalista, y con ello, en la transformación de todos los aspectos de la dinámica tradicional de la región (Mungaray, 1988).

Conestos señalamientos, se podría avanzar que las maquiladoras, 
más que una manifestación específica de lo que actualmente se ha dado en llamar como internacionalización del capital y por ende, de una nueva división internacional del trabajo, son una manifestación del fenómeno de extensión y homogenización capitalista a regiones marginales integradas al circuito de la economía mundial, a fin de integrarlas con mayor dinamismo a éste. Esto significaría una mayor ampliación del mercado mundial que amplía y reproduce el capital en su conjunto, tanto desde el punto de vista de la opcración de los grandes capitales, como de la incorporación asociada o independiente de los empresarios regionales a las actividades de producción y acumulación capitalista. Yendo más lejos, y en función de las tendencias observadas en la economía mundial que indican una profundización extrema de la división social del trabajo y una creciente automatización de los procesos productivos, las maquiladoras tal y como han existido tradicionalmente, muestran un carácter transitorio como instrumento de acumulación y reproducción del capital mundial, lo que obligará a losdefensores y críticos de esta modalidad de acumulación capitalista, a repensar sus estrategias y críticas de la industrialización regional vía maquiladoras $\mathrm{y}$, fundamentalmente, todo el cuerpo térico que anima su pensamiento y actuación.

\section{AGRADECIMIENTOS}

Este trabajo ha sido realizado entre 1983 y 1988 . Versiones no publicadas antes, se han presentado como conferencias en la Facultad de Economía de la Universidad Autónoma de Baja California (UABC), en noviembre de 1983 y en la escuela de Economía de la Universidad Autónoma de Nayarit (UAN), en abril de 1984, y como comentarios en el Simposium "Industrialization in Mexico", organizado por el Programa Chicano, de la Universidad de California, en Irvine, en mayo de 1986. Ello ha permitido su crítica y revisión constante. Se agradecen los comentarios de Fernando Jiménez (UABC), Julio Lopez (UNAM), y Javier Castellón (UAN) en 1984; Víctor Castillo (UABC), Alejandro Dabat (UNAM), y Luis SuárezVilla (UC Irvine) en 1986; Ricardo Pozas (COMECSO), y Esperanza Fujigaki (UNAM) en 1987; y de Arturo Ranfla (UABC) en 1988, así como los siempre agudos comentarios de mis alumnos en los Seminarios que sobre el tema he impartido en las facultades de Economía de la UNAM y la UABC. Por último, agradezco el apoyo financicro de 
la UABC, para terminar la investigación que se iniciara en las ideas de la primer versión de este trabajo, y el apoyo de Ma. de los Angeles Chavira, en su mecanografía. 


\section{B I B L I O G R A F I A}

ALVAREZ, Juan y Mungaray, Alejandro 1986. "Maquiladoras, política económica y medio ambiente en la frontera norte de México".En: Alvarez, Juan y Castillo, Víctor. Ecologia y Frontera/Ecology and The Borderlands, Tijuana, Escuela de Economía, UABC.

ALCALA Quintero, Francisco 1969. "Desarrollo regional fronterizo", Comercio Exterior, México, Banco Nacional de Comercio Exterior, vol. XIX, No. 12.

ANDERSON, Joan B. 1984, "Female participation and efficiency in mexican electronic and apparel assembly plants", Campo Libre. Joumal of Chicano Studies, Los Angeles, California State University, Los Angeles, vol. II, winter-summer. No. 1-2.

ANDERSON, Joan B. and Frantz, Roger S. 1985, " Reduction efficiency among mexican apparel assembly plants", The Joumal of Development Areas, Western Illinois University, april, No. 19.

APPLEYARD, Denis R., Field Jr., Alfred J. and Tower, Edward. 1985 "Further analysis of the effects of offshore assembly provisions on the US tariff structure", Joumal of Economic Studies, MCB. University Press, vol. 12, No. 4.

ARRIOLA Woog, Mario. 1981, El programa mexicano de maquiladoras. Una respuesta a las necesidades de la industria norteamericana, Guadalajara, Instituto de Estudios Sociales, Universidad de Guadalajara.

BAEZ Rodríguez, Francisco. 1983, "Maquiladoras c integración subordinada", Economia Informa, México, Facultad de Economía, UNAM, noviembre, No. 110.

BAIRD, Peter and Mc Caughan, Ed. 1975, Las maquiladoras en México, nueva lanza del Imperialismo, Oakland, North American Congress of Latin America.

BALLI, Federico, 1980, La industria de la maquila en México. Estudio monográfico, México, CEED, Colegio de México, Mimeo.

BANAMEX. 1979, Examen de la situación económica de México, México, diciembre, vol. LV, No. 649.

BANAMEX. 1981a, "The external sector", Review of the economic situation of México, México, may, vol. LVII. 
BERMUDEZ, Antonio. 1966, El rescate del mercado fronterizo, México, Eufesa.

BNCE (Banco Nacional de Comercio Exterior). 1978, "Industrias maquiladoras: evolución reciente y perspectivas", Comercio Exterior, México, abril, vol. XXVIII, No. 4.

BLANCO Mejía, José. 1983. "La maquila no puede ser base del desarrollo industrial", Economía informa, México, Facultad de Economía, UNAM, noviembre, No. 110.

BRIGGS, Vernon M. 1973, Foreign assembly industries in Mexico: a necessary evil of a underdeveloped society, Austin, Conference on economic relations between Mexico and the United States, april.

BRIONES, Alvaro. 1979,Economía politica del fascismo dependiente, México, Siglo XXI, eds.

BUSTAMANTE, Jorge. 1975, "El programa fronterizo de maquiladoras: observaciones para una evaluación", Foro Internacional, México, El Colegio de México, oct.-dic., vol. XVI, No. 2.

BUSTAMANTE,Jorge. 1976, Maquiladoras: a new face of international capitalism on Mexico's northem frontier, Atlanta, Sixth National Meeting of Latin American Association, march 23-24.

CALDERON, Ernesto. 1981. "Las maquiladoras de los países centrales que operan en el tercer mundo", Lecturas del CEESTEM-Maquiladoras, México, CEESTEM.

CARRILLO Huerta, Mario. 1978, "Desarrollo y crecimiento económicos: una interpretación", Ciencia Administrativa, Xalapa, IIESCA, Universidad Veracruzana, enero-junio, vol. I No. 1.

CARRILLO V.,Jorge. 1981, "Maquiladora clandestina en Tijuana cerró sus puertas", Boletín Informativo sobre Asuntos Migratorios y Fronterizos, México, Comité de Servicio de los Amigos, enero-febrero, No. 15.

CARRILLO V.,Jorge.1984a, "La internacionalización del capitaly la frontera México-Estados Unidos”, Investigación Económica, México, Facultad de Economía, UNAM, abril-junio, No. 168.

CARRILLO V., Jorge. 1984b, "Maquiladoras: industrialización fronteriza y riesgos de trabajo. El caso de Baja California", Economía: teoría y práctica, México, UAM, otoño. 
CARRILLLO V., Jorge y Hernández, Alberto. 1982a,: "Sindicatos y control obrero en las maquiladoras fronterizas", Investigación Económica, México, Facultad de Economía,UNAM, julio-septiembre, No. 161.

CARRILLO V., y Hernández, Alberto. 1982b, "Las maquiladoras en la frontera. Algunas corsideraciones para su evaluación". Iztapalapa, México, UAM.

CARRILlO V., Jorge y Hernández, Alberto. 1985, Mujeres fronterizas en la industria maquiladora, México, SEP CEFNOMEX.

CASTELLON F., F. Javier y Delgado L., Yolanda. 1985, "Los pasos perdidos. Origen y desarrollo de una planta maquiladora en Tepic,Nay." Convergencia, Tepic, Coordinación de investigación Científica, Universidad Autónoma de Nayarit, enero- junio, año 2 , No. 3

CHAVEZ, Eliza. 1981, "Las empresas matrices de la maquiladoras mexicanas". Lecturas del CEESTEM-Maquiladoras, México, CEESTEM.

CLEMENT, Norris C. 1987, "An overview of the maquiladora industry", en Paul Gangster (Ed.), The maquiladora program in trinational perspective: Mexico, Japan and the United States, San Diego Institute for Regional Studies of the californias, San Diego State University, (Border Issues Series, 2)

CLEMENT, Norris C. and Jenner, Stephen R. 1987, Location decisions regarding maquiladora/in-bond plants operating in Baja Califomia, Mexico, San Diego, Institute for Regional Studies of the Californias, San Diego State University, (Border Issues Series, 3).

COMITE DE SERVICIO DE LOS AMIGOS. 1980a, "Algunos datos recientes sobre maquiladoras en México", Boletin informativo sobre asuntos migratorios y fronterizos,México, junio-julio, No.12

COMITE DESERVICIO DE LOS AMIGOS. 1980b, "Las concxiones mundiales de las maquiladoras electrónicas", Boletín informativo sobre asuntos migratorios y fronterizos, México, octubre-diciembre, No. 14.

CORDERA, Rolando y Tello, Carlos. 1981, "Hacia un proyecto nacional", Nexos, México, abril, vol. 4, No. 40. 
DAVIS, Reginald L., 1985, Industria maquiladora y subsidiarias de co-inversión. Régimen juridico y corporativo, México, Cárdenas Ed.

DILlMAN C., Daniel. 1983, "Assembly industries in Mexico: contexts of development", Joumal of Interamerican studies and world affairs, Sage publications, february, vol. 25 No. 1.

DRUCKER, Peter. 1987, "La nueva economía mundial”, Contextos, México, SPP, segunda época, marzo, año 5, No. 76.

DUNCAN, Cameron. 1976, "The runaway shop in the mexican border industrialization program", Southwest economy \& society, october-december, vol. 2, No. 1.

ENRIQUEZ, Bill. 1971, The impact of the twin plants border commuter and ilegal entrans on the low-skill, low income american border resident, Manuscript, may 15.

ESCAMILLA, Norma y Vigorito, María Antonieta. 1979, "El trabajo femenino de las maquiladoras de ropa". Nueva Antropologia, México, No. 9.

FAJNZYLBER, Fernando. 1973, "La empresa internacional en la industrialización de América Latina”, en M.S. Wionczek (Ed.), Comercio de tecnología y subdesarrollo económico, México, UNAM.

FERNANDEZ, Raúl. 1980, La frontera México-Esțados Unidos. Un estudio socieconómico, México, Ed. Terra Nova.

FERNANDEZ, José Luis. 1980, "Algunos elementos que apuntan hacia un replanteamiento de la política de industrialización fronteriza", en varios,Las relaciones México-Estados Unidos, México, Ed. Nucva Imagen, vol. I.

FERNANDEZ, José Luis y De Buen, Bertha Elena. 1984, "Los límites del programa de industrialización fronteriza para el abatimiento de las tasas de desocupación y la calificación de la fucrza detrabajo", en Corona R. Alfonso y James Gibson Lay, (Comp.), Impactos regionales de las relaciones económicas México Estados Unidos, México, El Colegio de México.

FONEI. 1984, Programas generales de apoyo financiero, México, noviembre.

FROEBEL, Folker. Heinrichs, Jurgen y Kreye, Otto. 1978, "La nucva división internacional del trabajo. Sus origenes, sus manifestaciones, sus consecuencias", Comercio Exterior, México, BNCE, julio, vol. XXVIII, No. 7. 
FROEBEL, Folker., Heinrichs, Jurgen y Kreyc, Otto. 1981,La nueva división intemacional del trabajo, México, Siglo XXI eds.

GAMBRILL, Mónica Claire. S.F., Conciencia y organización en las maquiladoras, México, CES, El Colegio de México, Manuscrito.

GAMBRILL, Mónica Claire, 1979a, Composición y conciencia de la fuerza de trabajo en las maquiladoras, Monterrey, Simposio Nacional sobre estudios fronterizos, COLMEX-FFyL, UANL, enero 24 al 27.

GAMBRILL, M6nica Claire, 1979b, "Maquiladoras: el costo de sus trabajadores", Boletín informativo sobre asuntos migratorios y fronterizos, México, Comité de Servicio de los Amigos, julio-agosto, No. 7.

GAMBRILL, Mónica Claire. 1980, "El trabajo de las mujeres en la industria de la maquila", Boletín informativo sobre asuntos migratorios y fronterizos, México, Comité de Servicio de los Amigos, agosto-septiembre, No. 13.

GAMBRILL, Mónica Claire. 1981a, "La fuerza de trabajo en las maquiladoras. Resultados de una encuesta y algunas hipótesis interpretativas". Lecturas del CEESTEM, México, CEESTEM.

GAMBRILL, Mónica Claire. 1984, "Empleo vía maquiladoras: el caso de Tijuana", en Alfonso Corona R. y Lay James Gibson (Comp.), Impactos regionales de las relaciones económicas México-Estados Unidos, México, El Colegio de México.

GARCLA Moreno, Victor Carlos. 1979, La economía mexicana y la economía fronteriza del norte, Monterrey, Simposio Nacional sobre Estudios Fronterizos, COLMEX-FFyL, UANL, cnero 24 al 27.

GONZALEZ-Aréchiga, Bcrnardo. 1987, "Modernización industrial y crecimiento maquilador", El cotidiano, México, UAM, PCF SEP, COLEF, año 4, No. especial 1.

GONZALEZ R. Eduardo y López G., Julio. 1984, "Crisis y politica economica en el capitalismo desarrollado", El trimestre económico, México, FCE, enero-marzo, vol. LI (1), No. 201.

GRUNWALD, Joseph. 1983, "Reestructuración de la industria maquiladora", El trimestre económico, México, FCE, vol. L (4), oct.-dic.

GRUNWALD, Joseph. 1987, "U.S.-Mexican production sharing in world perspective", en Paul Gangster (Ed.) The maquiladoras program in trinational perspective: Mexico, Japan and the United States, San Diego, Institute for Regional Studies of the Califor- 
nias, San Diego State University, (Border Issues Series, 2). HANSEN, Niles. 1981, "México's border industry and the international division of labor", Annals of regional science, Washington, Western, Washington University, vol.XV, july, No. 2.

HARING, Henk A. 1985, Sunbelt frontier and border economy: manufacturing in El Paso-Ciudad Juárez, Utrecht, Departament of Geography, University of Utrecht (Utrechtse geografische studies 35 ).

HUNT, Lacy. 1970, "Desarrollo industrial en la frontera mexicana", Comercio exterior, México, BNCE.

IGLESIAS, Norma. 1984, La flor más bella de la maquila, México, SEP-CEFNOMEX.

IGLESLAS, Norma y Alarcón, Rafael. 1981, "Los standars de producción". Boletín informativo sobre asuntos migratorios y fronterizos, México, Comité de Servicio de los Amigos, enero febrero, No. 15.

IMCE (Instituto Mexicano de Comercio Exterior), México in-bond industry, México, enero.

JENSEN, Ronald W. and Flaherty, Colin. 1987, “U.S. -Japan trade war may divert business to México", Frontier business, San Dicgo, june-july.

JUAREZ, Antonio. 1979, Las corporaciones transnacionales y los trabajadores mexicanos, México, Siglo XXI eds.

KAMPETTER, Werner. 1979, Caracteristicas de las plantas maquiladoras. Hipótesis preliminares, México, CIDE, Mimeo.

KOEPP, Stephen. 1984, "Hands across the border". Time, september 10.

KONING, Wolfang. 1979, Efectos de la actividad maquiladora fronteriza en la sociedad mexicana, Monterrey, Simposio Nacional sobre Estudios Fronterizos, COLMEX-FFyL, UANL, enero 24 al 27.

KUESEL, Corinna. 1986, El papel de la industria maquiladora en el proceso de desarrollo e industrialización de México. Algunas hipótesis provocativas, México, Conferencia Frontera México - Estados Unidos. Chicanos, Pachucos y Cholos, 27 al 31 de octubre.

LEWIS, Arthur. 1975, La planeación económica, México, FCE. 
LOPEZ Lucio, Rogelio. 1985, La industria maquiladora de exportación y su relación con el desarrollo económico de Baja Califomia, Tijuana, Primer Foro Economico sobre Baja California, Colegio de Economistas de B. C. , julio 20.

MARTINEZ DEL CAMPO, Manuel. 1983, "Ventajas e inconvenientes de la actividad maquiladora en México. Algunos aspectos de la subcontratación internacional". Comercio exterior, México, BNCE, febrero, vol. 33, No. 2.

MARX, Karl. 1977a, Elementos fundamentales para la crítica de la economía política (Grundrisse), 1857-1858, México, Siglo XXI eds., 9a. ed., vol. 1.

MARX, Karl. 1977b, El capital. Contribución a la crítica de la economía política, México, Siglo XXI eds., 8 vols.

MINIAN, Isaac. 1981, Progreso técnico e intemacionalización del proceso productivo: el caso de la industria maquiladora de tipo electrónica, México, CIDE, (Series Ensayos, 2).

MÜLLER, Viana. 1976, Las condiciones socioeconómicas del trabajo en las maquiladoras desde la perspectiva de la investigación directa, México, El Colegio de México, Manuscrito.

MUNGARAY L., Alejandro. 1983a, "Maquiladoras, prueba de fuero", Economía informa, México, Facultad de Economía, UNAM, enero, No. 100.

MUNGARAY L., Alejandro. 1983b, "Division internacional del trabajo y automatización de la producción: el futuro de las maquiladoras", Investigación económica, México, Facultad de Economía, UNAM, abril- junio, No. 164.

MUNGARAY L., Alejandro. 1983c, "Maquiladoras, teoría y práctica", Economía informa, México, Facultad de Economía, UNAM, Noviembre, No. 110.

MUNGARAY L., Alejandro. 1985a, "Maquiladoras e infraestructura industrial en el norte de México, Diálogo, México, Banco $\mathrm{BCH}$, marzo-mayo, No. 18.

MUNGARAY L., Alejandro. 1985b, "La automatización de la industria norteamericana en el contexto de competencia internacional", Estudios fronterizos, Mexicali, Instituto de Investigaciones Sociales, UABC, Mayo-dic. vol. III, Nos. 7-8.

MUNGARAY L., Alejandro. 1988, "Maquiladoras y desarrollo industrial en la frontera norte de México". En Ricardo Pozas y Matilde Luna (Coords.) Las empresas y los empresarios en México, México, Grijalbo, en prensa. 
MUNGARAY L., Alejandro y Alvarez, Juan. 1987, "La oferta de espacios industriales en el desarrollo de Tijuana". FONEP, México, Fondo Nacional de Estudios y Proyectos, enero, No. 123.

MURAYAMA, Guadalupe y Muñoz, Ma. Elena. 1975, La mano de obra femenina en la industria maquiladora de exportación, México, CENIET, Mimeo.

NORRIS \& ELLIOT, S. A. de C. V. 1969, Survey on border development program, México, American Chamber of Com merce of Mexico, A. C., july.

OJEDA, Mario. 1980, El futuro de las relaciones entre México y Estados Unidos", en Carlos Tello y Clark Reynolds (Coords.), Las relaciones México-Estados Unidos, México, FCE. (Lecturas 43).

OPALIN, León, "Maquiladoras: desarrollo mundial", Excélsior, México, encro 31.

OPPENHEIM, Charles H. y Pedroza, Elba. 1987 “Maquiladoras: hacia una nueva frontera”. Expansión, México, abril 15, vol. XIX, No. 463.

ORTIZ Muñiz, Gilberto. 1986, "La industria maquiladora", Transformación, México, CANACINTRA, décima época, mayo, vol. III, No. 5.

PAI. 1986a, "La maquila de exportación", Pequeña y mediana industria, México, NAFINSA, enero-febrero, año 6, No. 51.

PASINETTI, Luigi L. 1980, Crecimiento económico y distribución de la renta, Madrid, Alianza Editorial.

PASINETTI, Luigi L. 1983, "Nicholas Kaldor: unas cuantas notas personales", Economía informa, México, Facultad de Economía, UNAM, No. 109, octubre.

PREBISCH, Raúl. 1980, "Prologo". en Rodríguez 1980.

POURDANAY, Nayereh. 1986, Les "Maquiladoras" et le developpement, París, ORSTOM, IEDES, noviembre, Mimeo.

PUENTE L., Sofía. 1986, "Las industrias maquiladoras en la frontera norte de México", Pequeña y mediana industria, México, NAFINSA, enero-febrero, año 6, No. 51.

REYES, Roberto. 1986, The mexican border experience on the implementation of the modern concept in industrial parks, Oklahoma, Economic Development Institute, The University of Oklahoma. 
REYNOLDS, Clark. 1980, "Las perspectivas económicas y sociales para México y sus implicaciones para las relaciones con los Estados Unidos", en Tello, Carlos y Reynolds, Clark (Coords.), Las relaciones México-Estados Unidos, México, FCE (Lecturas 43).

REYNOLDS, Clark W. 1985, "Industrial strategy and policy: Mexico and the United States complementation and conflict", Miranda, Marioy Wilkie, James (Eds.), Reglas del juego yjuego sin reglas en la vida fronteriza, México, ANUIES/PROFMEX.

RIVAS, Sergio F. 1985, "La industria maquiladora en México. Realidades y falacias", Comercio exterior, México, BNCE, noviembre, vol. 35 , No. 11.

RIVAS Sosa, E. 1973, Funciones de las industrias maquiladoras en la promoción de polos de desarrollo industrial, México, Facultad de Economía, UNAM, Tesis profesional.

RIVERA C., Héctor Arturo. 1971, La industria de la maquila en la frontera norte de México y el derecho intemacional, México, Facultad de Derecho, UNAM, tesis profesional.

RODRIGUEZ, Octavio. 1980, La teoría del subdesarrollo de la CEPAL, México, Siglo XXI, eds.

ROSADO Matos, Margarita. 1976, La condición de las obreras en las maquiladoras de la frontera nonte, México, El Colegio de México, Tesis profesional de Relaciones Internacionales.

SALAS-Porras, Alejandra. 1987, "Maquiladoras y burguesía regional", El cotidiano, México, UAM-PCF/SEP-COLEF, año 4 , número. especial 1.

SAVANE, Marie Angelique. 1980, "El empleo de la mujer, los cambios sociales y la liberación femenina. El caso de Africa", Comercio exterior, México, BNCE, agosto, vol. 30 , No. 8.

SIC (Secretaría de Industria y Comercio). 1975, Posibilidades del programa de maquiladoras, México, Subsecretaría de Industría.

SPP. (Secretaría de Programación y Presupuesto.) 1986, Estadística de la industría maquiladora de exportación 1974-1985, México, INEGI.

SMITH, Adam. 1979, Investigación sobre la naturaleza y causas de la riqueza de las naciones, México, FCE.

SKLAIR, Leslie. 1987, Does Mexico's maquiladora programme represent a genuine development strategy, La Jolla, Center for U.S.-Mexican Studies, UCSD, Mimeo. 
SPAETH, Anthony. 1979, "The maquila boom", Forbes, december 10. STEINER, Henry M. 1971, "Twin plants sprout along U.S. mexican border", Industrial development, Austin, july-august.

STORPER, Michacl and Walker, Richard. 1984, "The spatial división of labor and location of industries", en L. Sawer \& N. Tabb (Eds.), Sunbelt/snowbelt. The political economy of urban development and regional restructuring, New York-London, Oxford University Press.

SUAREZ Villa, Luis. 1982, "La utilización de factores en la industria maquiladora de México". Comercio exterior, México, BNCE, octubre, vol. 32 , No. 10.

SUAREZ Villa, Luis. 1984, "Technical dualism. The sunbelt and Mexico's border areas industrialization", en Corona R., Alfonso y James Gibson, Lay (Comp.), Impactos regionales de las relaciones económicas entre México-Estados Unidos, México, El Colegio de México.

SUAREZ Villa, Luis. 1985, "Urban growth and manufacturing change in the United States-Mexico borderlands: a conceptual framework and an empirical analysis", Annals of regional science, Washington Westerns, Washington University, november, vol. 19, No. 3 .

TALBOT, Cindy \& Hricko, Andrea. S.F., "Hazards of the electronic in industry",LOPH Monitor, Berkeley, Labor Occupational Health Program, Institute of Industrial Relations, University of California at Berkeley, special edition.

TAMAYO, Jesús. 1980, "Dos problemas del desarrollo económico fronterizo", en Varios, Las relaciones México-Estados Unidos, México, Ed. Nueva Imagen, vol. I.

TAMAYO, Jesús. 1985, "The northern border of Mexico and the crisis of 1982: a few preliminary observations",En James Gibson, Lay \& Corona R. Alfonso (Eds.), The U.S. and Mexico: borderland development and the national economies, Boulder and London, Westview Press.

TAMAYO, Jesús y Fernández, José Luis. 1983, Zonas fronterizas (México-Estados Unidos), CIDE México.

TEUTLI, Guillermo. 1976, "Las industrias maquiladoras, progreso y retroceso en el proceso mexicano de desarrollo", El economista mexicano, México, Colegio Nacional de Economistas, julio-agosto, vol. XI, No. 1. 
TEUTLI, Guillermo. 1981, "La industria maquiladora como gencradora de divisas y empleo", El economista mexicano, México, Colcgio Nacional de Economistas, mayo-junio, vol. $\mathrm{XV}$, No.3

TORO, Ramón del. 1979, "Un modelo del esquema desarrollado en la frontera norte", El economista mexicano, México, Colcgio Nacional de Economistas, marzo-abril, vol. XIII, No. 2.

TORRES, Olga Ester. 1976, "Algunas observaciones sobre la economía de la frontera norte", Comercio exterior, México, BNCE, diciembre, vol. 26, No. 12.

TORRES Gaytán, Ricardo. 1979, "Intercambio, comercio y desarrollo de la zona fronteriza norte". El economista mexicano, México, Colegio Nacional de Economistas, julio-agosto, vol. XIII, No. 4.

TRA.JTENBERG, Raúl. 1978, Transnacionales y fuerza de trabajo en la periferia. Tendencias recientes en la internacionalización de la producción, México, ILET.

URQUIDI, Víctor y Méndez V., Sofía. 1975, "Importancia econòmica de la zona fronteriza del norte de México", Foro internacional, México, El Colegio de México, octubre diciembre, vol. XVI, No. 2.

VAITSOS, Constantine. 1979, "Poder,conocimiento y política de desarrollo: relaciones entre las empresas transnacionales y los países en desarrollo", G. K. Helleiner (Ed.), Hacia un nuevo orden económico intemacional?. México, Siglo XXI eds.

VALDEZ Cota, María Ramona. 1985, "Impacto de las devaluaciones del peso de 1976 y 1982 en las maquiladoras de Baja California". Economía informa, México, Facultad de Economía, UNAM, abril, No. 127.

VERA, Beatríz E. 1980, "El impacto del trabajo asalariado en la vida de las mujeres". Boletín informativo sobre asuntos migratorios y fronterizos, México, Comité de Servicio de los Amigos, abril-mayo, No. 11.

VERKOREN, O. y Hoenderdos W. 1986, La politica industrial en México y la industrialización en la zona fronteriza del norte de México, Utrecht, Instituto de Geografía, Universidad Nacional de Utrecht, Mimeo.

VILLARESPE, Verónica y Rodríguez, Salvador. 1981, El proceso de trabajo en las maquiladoras de exportación. El caso de Crescent-Zacatecas, $S$. A., México, 2o. Seminario Internacional 
sobre Crisis, Nuevas Tecnologías y Procesos de Trabajo, DEPFE y DEPFCPyS-UNAM, julio 20 al 30.

VILLAREAL, René. 1983, La contrarrevolución monetarista, México, Ed. Océano.

VILLAREAL, Roberto. 1987, La industria maquiladora y el desarrollo económico de la frontera norte, México, Unidad de la Crónica Presidencial, Mimeo.

XIRAO Icaza, Joaquín y Díaz, Miguel. 1976, Nuestra dependencia fronteriza, México, FCE (Archivo 48). 